\title{
Changes in Endogenous Gibberellins in the Maturing Fruits of Watermelon*
}

\author{
by Prithvi R. BHALlA**
}

Received July 3, 1970

\begin{abstract}
The fruits of watermelon, Citrullus lanatus were collected at different stages of development from plants grown at the bank of river Jamuna, Delhi, to investigate the changes in gibberellins during their progressive maturation. Gibberellinlike substances in fruits were detected at all stages, namely, 5, 7, 9, 1., 13, 14, 17,25 and 35 days after pollination. The activity as bioassayed by $\mathrm{d}_{2}$ mutants of Zea mays was very low 5 days after pollination, but rose to a peak on the 17 th day and declined as the fruit matured. The maximum activity was obtained in the fruit when it attained only $40 \%$ of its final fresh weight which indicates that the gibberellin-like factors, detected in the present work, may have a role in fruit growth. Chromatography of the acidic fraction of the pulp of mature watermelon fruits using different solvents provides evidence for the presence of at least three gibberellin-like factors.
\end{abstract}

Endogenous gibberellin-like substances have been demonstrated in the fruits of watermelon $^{1)}$ as well as in several other fruits ${ }^{2-7)}$. However, there are only a few investigations known to the author concerning the changes of concentration of gibberellin-like substances in relation to the fruit development ${ }^{2,5,6)}$. Further studies on this aspect should extend our understanding of the physiology of fruit and seed development. The present investigation, therefore, deals with the extraction and estimation of type and quantity of gibberellins in the fruits of watermelon botanically known as Citrullus lanatus during their progressive maturation.

\section{Materials and Methods}

For initial studies commercially available mature watermelon fruits were used. Subsequently, fruits at different stages of development were obtained from plants grown in a field on the bank of river Jamuna in Delhi, India.

The watermelon is an annual plant cultivated in northern India during summer. Three or four varieties of watermelon are commonly grown in Delhi. Although no definite nomenclature exists, these are easily distinguished by farmers by their fruits which vary in size, shape, colour and sweetness of the pulp. In the present investigation, a variety with large fruits and light green rind was used. The seeds are sown during the last week of January and the first week of February. The plant starts fiowering towards the end of March. Each plant generally bears 12-15

* The material of this publication is from $\mathrm{Ph} . \mathrm{D}$. thesis approved by University of Delhi, India.

** Department of Botany, University of Kentucky, Lexington, Kentucky 40506, U.S.A. 
flowers, durings its life span, of which 7 or 8 may be male, 3 or 4 female and 2 or 3 bisexual. Eventually, only one or two flowers, however, give rise to mature fruits.

Since the present investigation was undertaken to determine the changes in gibberellin-like substances during progressive maturation of watermelon fruits, it was considered essential to determine the growth curves of the fruit.

The growth of fruit, as measured by its volume, is nearly log-linear 7-10 days after pollination and thereafter it is comparatively slower. However, the fruits take about 35 days to mature and attain their maximum weight.

Determination of the rate of fruit growth-The flowers were tagged in the field to mark the day of pollination. Every second or third day, after pollination had taken place, the length- and breadthwise circumferences of the enlarging fruits were measured with the help of a thread and rule. From the average of these circumferences, the volume of the fruit was calculated employing the formula: volume $=(\text { circumference } / 2 \times 3.14)^{3} \times 4.189 . \quad$ An age-volume relationship was then plotted and from this curve the approximate age of any fruit could be determined. The growth rate of fruits was measured also by taking fresh weights regularly in the field.

The watermelon fruits, of different ages, were excised from healthy plants and brought to the laboratory. Seeds were separated from the pulp with the help of a needle or a pair of forceps. The pulp was homogenized and immediately stored in a deep freeze at $-25^{\circ}$.

Extraction and purification procedure-Pulp, about $500 \mathrm{ml}$, was taken out and thawed at room temperature. It was filtered and the residue extracted twice with approximately $250 \mathrm{~m} l$ acetone: water $(1: 1)$, followed two times each with similar quantities of methyl and ethyl alcohol. The aqueous watermelon filitrate and the extracts were combined for further processing.

The organic solvent/s were distilled off in a flash evaporator at $40-50^{\circ}$ leaving behind an aqueous concentrate. The $\mathrm{pH}$ of this solution was adjusted to 2.5 with $1 \mathrm{~N} \mathrm{H}_{2} \mathrm{SO}_{4}$. The solution was stirred with activated charcoal $(20 \mathrm{~g} / l)$, the charcoal filtered, and then eluted with 10 volumes of $70 \%$ acetone. The charcoal treatment and elution was repeated four times. The acetone was distilled off under vacuum at $40-50^{\circ}$ and the residual aqueous solution treated with basic lead acetate (about $10-15 \mathrm{~g}$ per $100 \mathrm{ml}$ ). The mixture was centrifuged at low speed and the precipitate discarded. The supernatant was then adjusted to $\mathrm{pH} 7.5$ with $10 \%$ sodium bicarbonate and extracted four times with equal volume of ethyl acetate. This ethyl acetate or the 'neutral' fraction was dehydrated over anhydrous sodium sulphate, evaporated to dryness and the residue tested for activity on a bioassay. The waterphase was acidified to $\mathrm{pH} 2.5$ with $1 \mathrm{~N}_{2} \mathrm{SO}_{4}$ and extracted four times with equal volumes of ethyl acetate. The second ethyl acetate fraction or the so-called 'acidic fraction', provided most of the material for the present studies. The 'acidic' and 'neutral' fractions were further purified by paper, column, or thinlayer chromatography or a combination of all these techniques.

Paper chromatography-Whatman paper no. 1 was used for descending chromatography. The solvents employed are mentioned in the text.

Celite partition chromatography-The acidic fraction was purified by celite partition chromatography according to the method of Kende and Lang ${ }^{88}$.

Thin-layer chromatography-The method described by MacMillan and Suter ${ }^{9)}$ was followed. The qualitative analysis of standard gibberellins, applied simultaneously, was done by ethanol: conc. sulphuric acid $(95: 5)$ spray. The thin-layer 
chromatograms were then heated for 10 minutes at $110^{\circ}$ and examined under ultraviolet light. The gibberellins are visible as blue, purple and yellow fluorescent spots.

Bioassays-The main bioassay systems were those employing dwarf corn mutants $\left(d_{1}\right.$ and $\left.d_{2}\right)$ and dwarf pea. The dwarf corn bioassay was done as described by Phinney and West ${ }^{10)}$. If the growth of the treated seedlings exceeded that of controls by more than $25 \%$, it was taken as an evidence for the presence of gibberellin-like activity.

The dwarf pea bioassay was done according to the method of Kende and Lang ${ }^{8)}$ using seeds of Pisum sativum var. Progress no. 9 (obtained from Ferry-Morse Seed Co., Mountain View, Calif., U.S. A.). The increase in growth of treated plants over controls was taken as a measure of gibberellin-like activity.

The quantitative estimation of gibberellin-like activity (in terms of $\mathrm{GA}_{3}$ equivalents) was done with the aid of a standard curve, the data for which were obtained by application of known quantities of gibberellic acid $\left(\mathrm{A}_{3}\right)$ on corn or pea seedlings.

\section{Results}

Quantitative changes in gibberellin-like activity in the developing fruits.

The pulp from fruits was analyzed for gibberellin-like substances at nine stages of development, namely, 5, 7, 9, 11, 13, 14, 17, 24 and 35 days after pollination, covering almost the entire development period. The crude acidic fraction of the pulp was purified by thin-layer chromatography. The $d_{2}$ mutants of maize were used for bioassay of gibberellin-like substances. Fig. 1 combines the results of 9 separate experiments undertaken to locate gibberellin-like activity at different stages of development. A general glance shows that there are three zones of activity corresponding to $\mathrm{Rf} 0-0.4,0.5-0.7$, and $0.8-1.0$ indicating that there may be at least 3 different active factors. The main activity was located at $\operatorname{Rf} 0-0.4$; at the two other zones, the activity was almost equally distributed.

Fig. 2 represents the total activity between $\mathrm{Rf} 0-0.4$ extractable by the present technique. This was accomplished with the aid of the standard curve relating the growth of $\mathrm{d}_{2}$ mutants of maize to doses of gibberellic acid. Gibberellin-like activity was very low 5 days after pollination, being of the order of $0.002 \mu \mathrm{g} \mathrm{GA}_{3}$ equivalents/ $\mathrm{g}$ fresh weight. It gradually increased to $0.33 \mu \mathrm{g} \mathrm{GA}_{3}$ equivalent/g fresh weight on the 17th day after pollination when the fruit attains a volume of about 4 litres. The total quantity of gibberellin-like substances in a fruit at this stage was calculated to be approximately $1.15 \mathrm{mg} \mathrm{GA}_{3}$ equivalent. The amount then declined and was $0.8 \mathrm{mg} \mathrm{GA}_{3}$ equivalent/fruit as maturation proceeded. The gibberellin-like activity, on the basis of dry weight, followed a rise and fall pattern essentially similar to that on the basis of fresh weight.

Comparison of GA-activity with the growth of the fruit reveals that the peak is attained after the most active period of growth. The pulp of the fruit, at this stage, is already slightly pinkish in colour. The seeds embedded in the pulp, also attain their maximum size by this time. The embryos show well-differentiated cotyledons whereas the endosperm is almost degenerated. Apparently, the rate of growth of fruits and the total amount of gibberellin-like substances in the pulp are not too closely related. This matter is taken m. again under "Discussion'. 


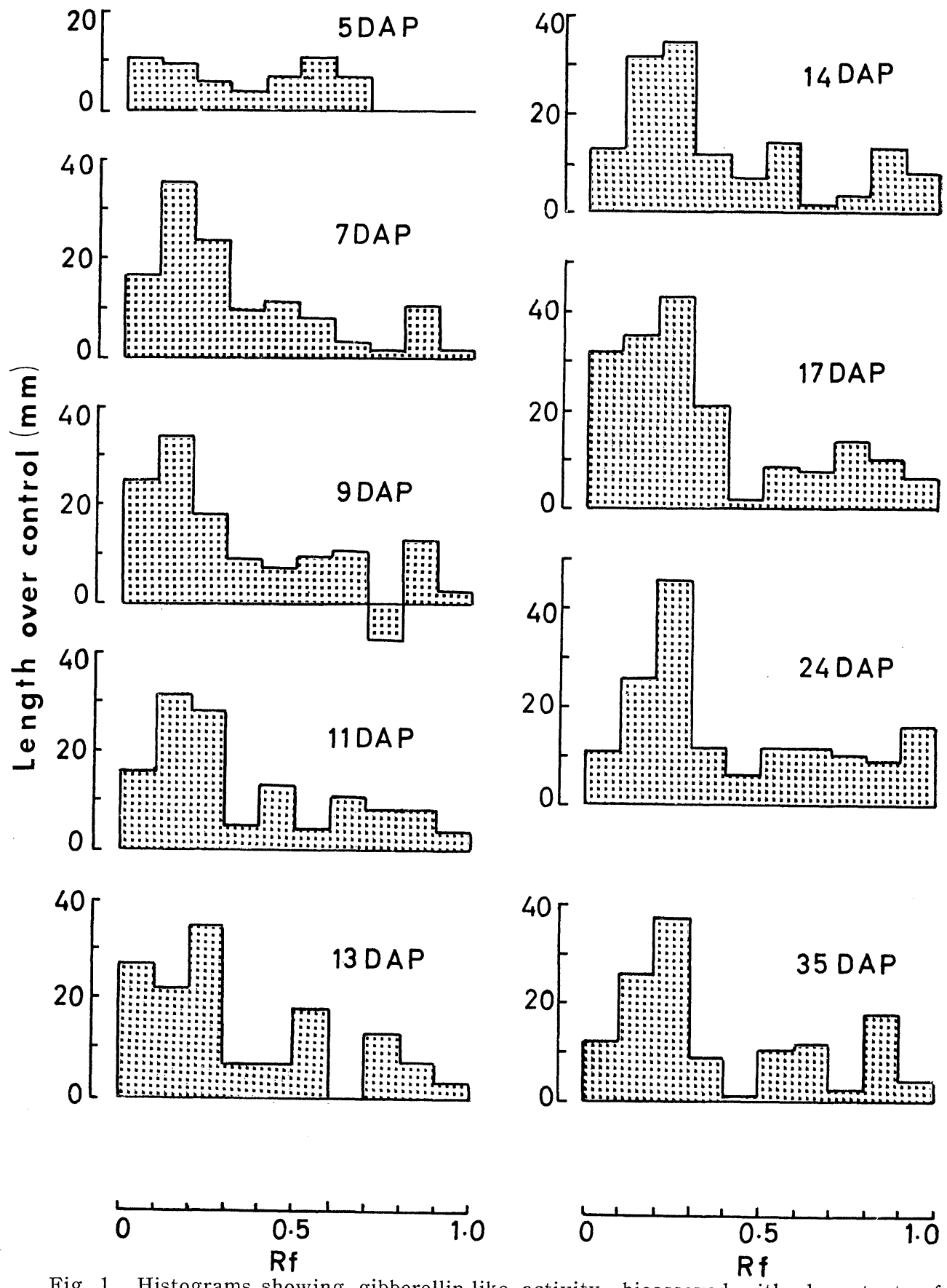

Fig. 1. Histograms showing gibberellin-like activity, bioassayed with $\mathrm{d}_{2}$ mutants of Zea mays, from the pulp of the watermelon fruits during their progressive maturation. The extract was purified by thin-layer chromatography using di-isopropyl ether : acetic acid (95:5) as solvent. The chromatogram was divided into 10 portions and tested for activity after elution. The Rf values appear on the abscissa and growth over control has been plotted on the ordinate. DAP represents days after pollination. 


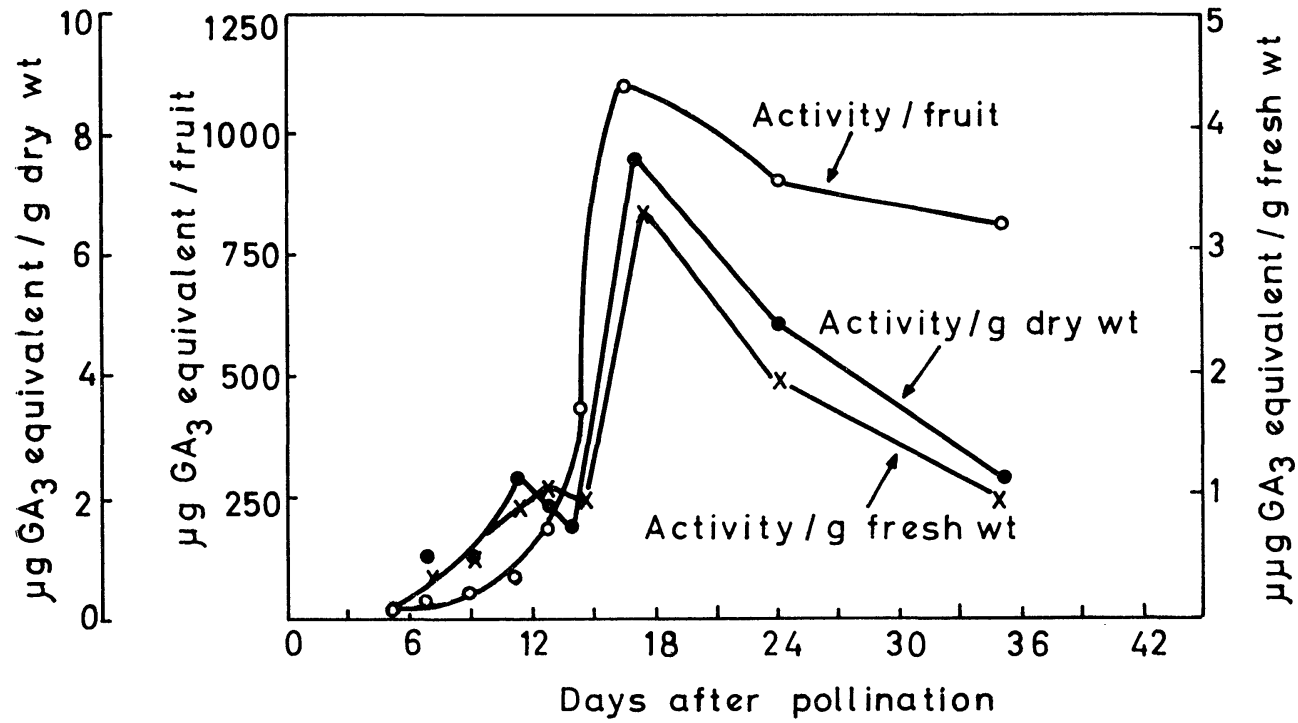

Fig. 2. Changes in the amount of gibberellin-like materials at different stages of fruit development. Extracts were assayed for activity on $\mathrm{d}_{2}$ mutants of Zea mays. Response in bioassay was converted to gibberellic acid equivalents by means of the dosage-response curve.

\section{Qualitative analyses of gibberellin-like factors in mature watermelon fruits}

Table 1 summarizes the various chromatographic solvents used to separate gibberellin-like substances from the pulp and the results obtained.

Chromatographic purification of the acidic fraction of the juice on Whatman no. 1 parper, using isopropanol: ammonia: water $(10: 1: 1)$, revealed two active zones. These corresponded to $0.1-0.2$ and $0.3-0.9$. The major activity was distributed at Rf 0.4-0.7. When $n$-butanol: $1.5 \mathrm{~N}^{2} \mathrm{NH}_{4} \mathrm{OH}(3: 1)$ was used as the chromatographic solvent, two active regions were observed at $\mathrm{Rf} 0-0.3$ and $0.6-0.7$. The eluates of segments at Rf $0.3-0.4$ and $0.7-0.9$ inhibited growth of test seedling.

When water was used as the solvent, two peaks of activity at Rf $0-0.2$ and Rf 0.7-1.0 were observed (Fig. 3A). An essentially similar distribution of activity was recorded when the dwarf pea bioassay was used (Fig. 3B). However, eluates of the chromatogram segments at $\mathrm{Rf} 0.3-0.5$ caused injury to the test plants, probably because of growth inhibitors, present. Rechromatography of the eluates of Rf 0.51.0 , of the chromatogram developed with water, using $n$-butanol : $1.5 \mathrm{~N} \mathrm{NH}_{4} \mathrm{OH}(3: 1)$ as the solvent, divided the activity between two zones: one at $\operatorname{Rf} 0.1-0.4$ and the other at 0.5-0.6 (Fig. 4). These results indicate that there are at least three gibberellin-like substances present in the juice of mature watermelon fruits.

Thin-layer chromatography of the crude acidic fraction on plates coated with silica gel $G$ and developed with di-isopropyl ether: acetic acid (95:5) revealed three zones of activity at Rf $0-0.1,0.2-0.5$ and $0.7-1.0$ in the dwarf pea bioassay. When benzene: acetic acid: water $(8: 3: 5)$ was used as the solvent system, three zones corresponding to Rf values $0-0.1,0.2-0.4$ and $0.6-0.8$ were observed. The major activity was present at Rf $0-0.1$.

The crude acidic fraction was also purified on buffered celite column. Bioassay with the $d_{2}$ dwarf maize mutant revealed gibberellin-like activity between fractions 


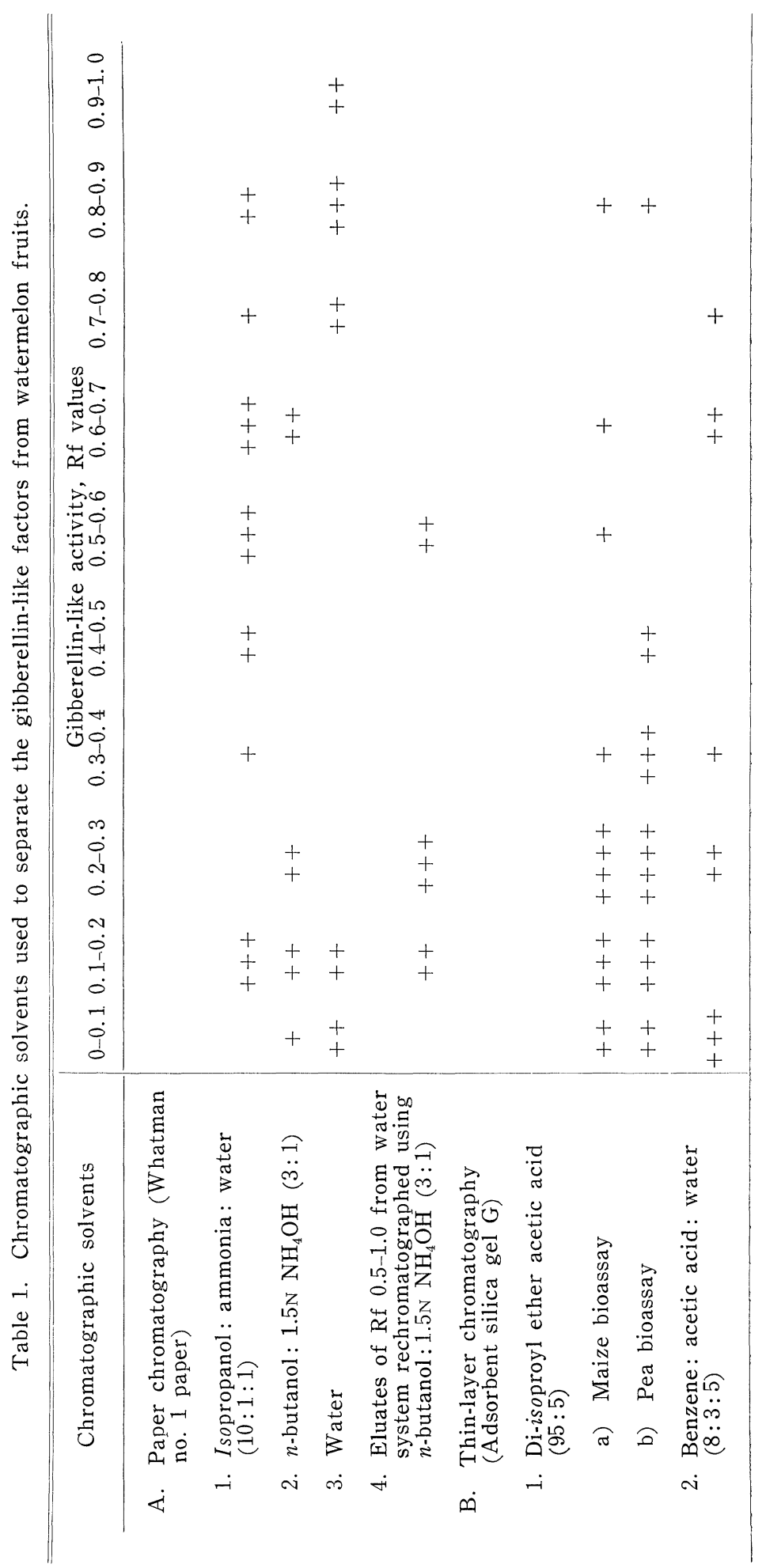



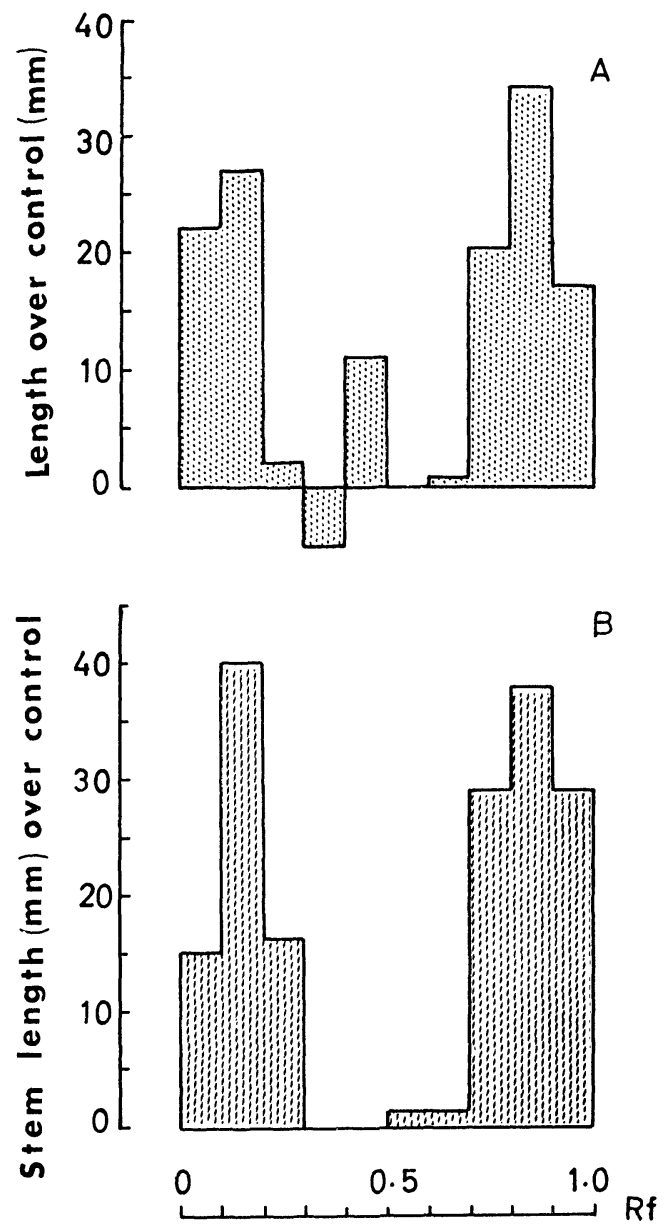

Fig. 3. Gibberellin-like activity by $\mathrm{d}_{2}$ mutants of maize (Fig. A) and dwarf pea (Fig. B) after paper chromatographic purification using water as the solvent.

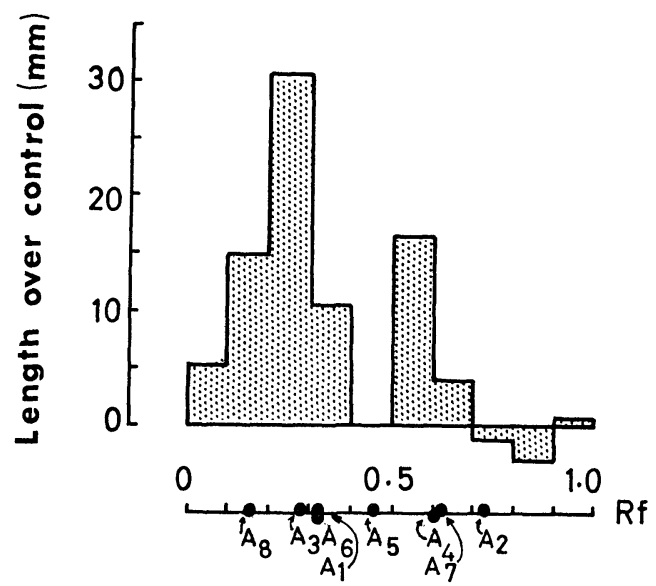

Fig. 4. Gibberellin-like substances in the eluates of the segments corresponding to Rf $0.5-1.0$ of paper chromatograms run with water and rechromatographed using $n$-butanol: $1.5 \mathrm{~N}$ ammonia $(3: 1)$ as the solvent. The relative mobilities of standard gibberellins are after MacMillan et al. ${ }^{9)}$

nos. 3 and 5, and in fraction no. 9. Essentially, a similar distribution of activity was observed when the dwarf pea bioassay was used.

\section{Discussion}

\section{Gibberellin-like substances and the growth of watermelon fruits}

The growth of several fruits in response to gibberellic acid suggest that gibberellin-like substances, pro-

duced by the seed, may also be controlling fruit development. Sachar and Kapoor ${ }^{11)}$ who treated the unpollinated ovaries of Zephyranthes with gibberellic acid obtained parthenocarpic fruits with seeds that were normal looking but devoid of embryos. Untreated ovaries failed to develop and aborted. Beside this and similar other reports, the relation between the production of gibberellin and growth of fruits is, however, even less clear than in case of seed growth. Most workers have studied only seeds, there being relatively little imformation concerning the changes in concentration of endogenous gibberellin-like substances during fruit development ${ }^{2,5,7)}$.

The present investigation has demonstrated the presence of gibberellin-like materials in developing watermelon fruits. The gibberellin-like substances are low during the early phase of fruit growth, but increase gradually until 10-12 days after pollination; the peak is found 17 days after pollination when the fruit has attained only $40 \%$ of its final fresh weight (Fig. 5). The amount declines thereafter 


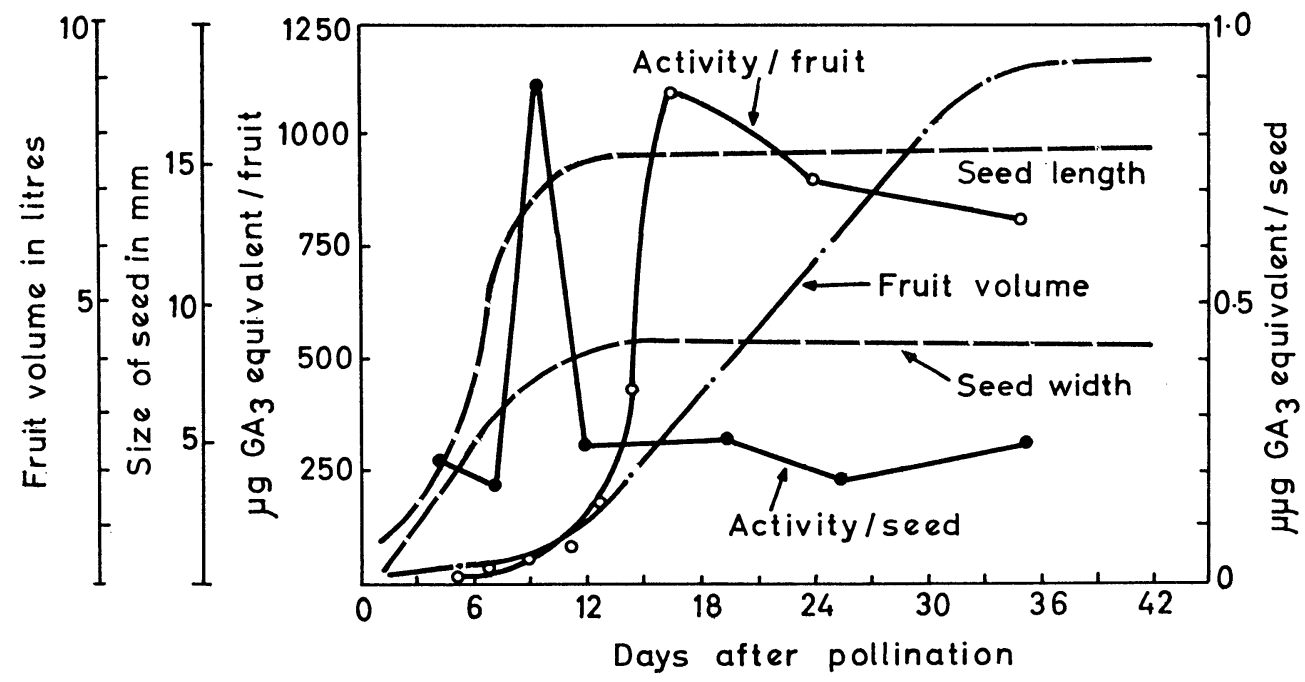

Fig. 5. Changes in the amount of gibberellin-like substances in watermelon fruits and seeds in relation to fruit and seed development. Activity/seed is after Bhalla ${ }^{12)}$.

and mature watermelon has low levels of gibberellin-like materials. These results show some connection between the content of gibberellin-like materials in watermelon fruits and their growth. These results are in conformity with the investigations of Jackson and Coombe ${ }^{5)}$ who found that the concentration of gibberellinlike factors in endosperm and mesocarp of the fruit of Prunus armeniaca correlates well with the growth of the fruits. Raju and Das ${ }^{7)}$ also observed that gibberellinlike substances in seeds of Capsicum annuum showed two definite peaks in contents which preceeded the maximal growth and maturation of the fruit.

Two separate peaks of gibberellin-like activity were found for the seeds and pulp of the watermelon fruits (see Fig. 5). In the seeds the concentration of the active factors is highest $9-10$ days after pollination (see Bhalla) ${ }^{12}$, whereas in the pulp it is maximum 17 days after pollination. Although it is possible that the growth of the fruit is initially regulated by gibberellin-like materials diffusing from seeds, at later stages the fruit appears to be self sufficient in the factors necessary for its growth. The peaks in the fruits and seeds are a little too far apart to make a simple translocation hypothesis valid and it appears that the pulp synthesizes its own gibberellin-like factors.

Identity of gibberellin-like factors in watermelon fruits

By repeated chromatography, watermelon fruits have shown to have three active gibberellin-like substances (see Table 1). On paper chromatograms run with $n$-butanol: $1.5 \mathrm{~N}$ ammonia $(3: 1)$, the activity was found to be distributed between Rf $0-0.3$ and $0.6-0.7$ (Fig. 4). The activity at $\mathrm{Rf} 0-0.3$ can be explained by the presence of either gibberellin $A_{1}$ or $A_{3}$ or a combination of both (Rf values according to MacMillan et al. ${ }^{11)}$ are 0.31 and 0.29 respectively). The second region of activity at 0.6-0.7 may be due to gibberellin $\mathrm{A}_{4}$ and $\mathrm{A}_{7}$ (reported $\mathrm{Rf}$ values are 0.6 and 0.61 respectively by MacMillan et $\left.a l .{ }^{11)}\right)$. These experiments show that there is at least one polar and one non-polar gibberellin-like factor present in the pulp of watermelon fruits. 
On the thin-layer chromatogram using di-isopropyl ether: acetic acid $(95: 5)$ and the maize bioassay, the major activity was found to be distributed between $\mathrm{Rf} 0$ 0.4 , the peak being at $\operatorname{Rf} 0.2-0.3$ in all but youngest stages analyzed. At younger stages the peak was located at Rf $0.1-0.2$ indicating that gibberellin $A_{1}$ or $A_{3}$ or both are present. Using the same solvent system but with the pea bioassay three peaks of activity between $0-0.1,0.2-0.5$, and $0.7-1.0$ were found. The activity at Rf 0.3-0.5 may be because of any combination of gibberellins $A_{4}, A_{5}, A_{7}$ (Rf values of gibberellins $A_{4}, A_{5}, A_{7}$ are $0.37,0.31$ and 0.37 respectively). Essentially similar results were obtained in another solvent system namely benzene: acetic acid: water $(8: 3: 5)$. In this solvent three regions of activity at $0-0.1,0.2-0.4$ and $0.6-0.8$ were observed. The activity at $0-0.2$ can be explained because of the presence of any or the combination of gibberellin $A_{3}$ and $A_{1}$, at 0.2-0.3 due to a unknown substance and at Rf 0.6-0.8 due to nonpolar gibberellin like $A_{7}$.

To conclude, on the basis of the chromatographic evidence, the watermelon fruits are shown to have at least three active gibberellin-like factors. The final identification of these active factors must await further purification employing quantities of materials larger than has hitherto been possible and to study their chemical and biological properties.

It is a pleasure to thank Dr. S. C. Maheshwari (Department of Botany, University of Delhi, India) for his stimulating advice and continual encouragement and Professor B. O. Phinney (University of California, Los Angeles, U. S. A.) for supplying seeds of dwarf mutants of maize.

\section{References}

1) Maheshwari, S.C. and Bhalla, P.R., Naturwissenschaften 53 : 89 (1966).

2) Coombe, B. G., Plant Physiol. 35: 241 (1.960).

3) Weaver, R. J. and Pool, R.M., Plant Physiol. 40 : 770 (1.965).

4). Dennis, F.G., Jr. and Nitsch, J.P., Nature 211: 781 (1966).

5) Jackson, D. I. and Coombe, B. G., Science $154: 277$ (1.966).

6) Iwahori, S. et al., Plant Physiol. 43: 333 (1.968).
7) Raju, P.V. and Das, V.S.R., Z. für Pflanzenphysiologie 58 : 266 (1.968).

8) Kende, H. and Lang, A., Plant Physiol. 39 : 435 (1.964).

9) MacMillan, J. and Suter, P.J., Nature 197: 790 (1.963).

10) Phinney, B. O. and West, C.A., Ann. Rev. Plant Physiol. 11: 441. (1960).

11) MacMillan J. et al., Adv. Chem. Ser. $28: 18$ (1.961).

12) Bhalla, P.R., Ph. D. Thesis, University of Delhi, India (1.967). 